\title{
Autoantibodies, autoimmune disease, and the birth of immune diagnostics
}

\author{
Eng M. Tan
}

The Scripps Research Institute, La Jolla, California, USA

\begin{abstract}
The appearance of autoantibody to DNA followed sequentially by the disappearance of anti-DNA and appearance of DNA antigen in a patient with systemic lupus erythematosus demonstrated that autoantibodies participate in immune complex-mediated pathogenesis. Continuing studies showed that autoantibodies are also useful biomarkers in clinical diagnosis and important reagents for elucidating the structure and function of intracellular proteins in cell biology. Recently, autoantibodies to tumor-associated antigens have been identified in cancer, and these findings have expanded the field of cancer immunodiagnostics.
\end{abstract}

In 1964, a young man with systemic lupus erythematosus (SLE) presented at the hospital of Rockefeller University in New York City with an acute exacerbation of his illness. This was brought on by his falling asleep under a sunlamp. He had a generalized erythematous sunburn rash, joint pain, high fever, and markedly increased proteinuria (1). He was hospitalized, treated with large doses of prednisone, and, after several weeks, went into remission (Figure 1A). Serum specimens had been obtained during his previous outpatient visits when his illness was relatively stable, and more specimens were collected during his hospitalization. These serial serum specimens were examined by double diffusion analysis in agarose for antibodies to DNA. The presence of antibodies was indicated by the appearance of immunoprecipitin lines (Figure 1B).

Immunologists in the 1950s and early 1960s had shown in experimental animals that antigen-antibody complexes produced vasculitis, including glomerulonephritis $(2,3)$, and the search was on for evidence of this form of pathogenic mechanism in autoimmune diseases. In the case of the SLE patient, six serum samples, collected on different dates, were set up against a central well containing solubilized DNA. Serum samples obtained before the episode of sustained high fever (February 14 and 19, 1964 [2-14 and 2-19]) contained precipitating antibody to DNA, but the antibody could not be detected in sera collected during disease

Conflict of interest: The author has declared that no conflict of interest exists.

Citation for this article: J Clin Invest. 2012; 122(11):3835-3836. doi:10.1172/JCI66510. flare (4-18 and 4-26). When this same set of samples was reacted against the pre-flare serum (from 2-19) in the central well, those obtained during disease flare (4-18 and 4-26) demonstrated precipitin reactions against factors in the serum of 2-19 (Figure 1B, lower set of wells). It was shown in other experiments that the sera of 4-18 and 4-26 contained DNA antigen and that the precipitin lines were DNA-anti-DNA reactions and were capable of fixing complement. These observations confirmed the presence of immune complex disease in autoimmunity and that autoantibodies to cellular antigens recognized as non-self by the immune system were involved. The sequential presence of antibody, absence of detectable antigen or antibody in the 3-26 specimen, and appearance of antigen would be in line with the immunochemistry of antigen-antibody reactions in solution related to phases of antibody excess, antigen-antibody equivalence, and antigen excess, respectively.

These studies were conducted while I was in the laboratory of Dr. Henry G. Kunkel (1916-1983) of Rockefeller University. The University Hospital was funded by a General Clinical Research Center grant from the NIH, and patients were often hospitalized for several weeks for extended immunological studies. For investigators, those were the halcyon days of clinical research.

\section{Autoantibodies as diagnostic biomarkers and reagents in cell biology}

The use of immunofluorescence techniques, in which tissue culture cells were used as antigen substrate for detecting autoantibodies, led to the detection of autoantibod- ies in various autoimmune diseases, including SLE, scleroderma, dermatomyositis, and mixed connective tissue disease. Certain autoantibodies produced distinct patterns of staining because they reacted with specific organelles in the nucleoplasm, nuclear membrane, nucleolus, or cytoplasm. An outstanding example is an autoantibody in the CREST (calcinosis, Raynaud's phenomenon, esophageal dysmotility, sclerodactyly, telangiectasia) subset of scleroderma, in which immunostaining revealed a limited number of dots in the nucleoplasm of interphase cells, but a total redistribution of these dots to the centromeric regions of condensed chromosomes of cells in mitosis. It became clear that there were multiple autoantibodies of different specificities in any individual autoimmune disease, a few autoantibodies were disease specific, and different autoantibody profiles were associated with different diseases (see review in ref. 4). Such profiles of autoantibodies now serve as diagnostic biomarkers in autoimmune diseases.

In addition to serving as disease markers, autoantibodies are also useful cell biology reagents (5). There was great effort by cell biologists to purify small nuclear ribonucleoprotein (snRNP) particles because of evidence that they were involved in the processing of precursor mRNA. When anti-Sm was detected in SLE (6), it led to the identification of anti-nuclear RNP. Both autoantibodies were used to immunoprecipitate snRNP particles and helped to elucidate their role in splicing pre-mRNA (7). Similarly, anti-centromere autoantibody (8) was used to study the structure and function of kinetochores, an organelle crucial to chromosome condensation and duplication in cell division (9).

\section{Autoantibodies as reporters identifying cellular factors involved in tumorigenesis}

The immune system is capable of sensing aberrant cellular factors that initiate tumorigenesis by making autoantibodies to tumor-associated antigens (TAAs). In a 
A

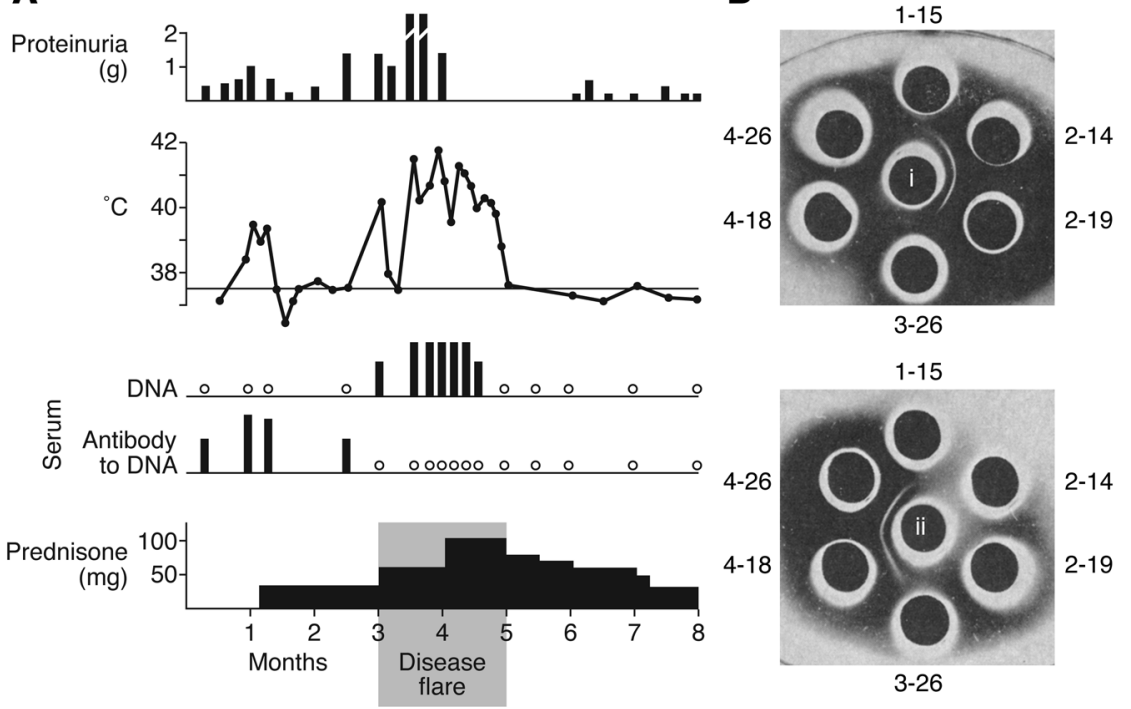

Figure 1

Identification of DNA antibodies in a patient with systemic lupus erythematosus. A patient with SLE experienced an acute exacerbation of illness with continuous daily fever of $40^{\circ} \mathrm{C}$ to $42^{\circ} \mathrm{C}$ for several weeks with increased proteinuria $(\mathbf{A})$. Sequential serum samples showed the presence of autoantibody to DNA, which was replaced by circulating DNA antigen during the disease flare. (B) Immunodiffusion studies demonstrating that pre-flare serum samples from 2-14 and 2-19 contained antibody to DNA (i, upper set of wells). In the lower set of wells, the central well was filled with serum from 2-19 (ii, containing autoantibody to DNA). Precipitin reactions were observed between this serum and those of 4-18 and 4-26. Chemical studies confirmed the presence of DNA in the latter two samples. Absence of either antibody to DNA or DNA antigen was observed at 3-26, a period of transition from antibody excess to antigen excess. The time line in A shows a period of eight months in the patient's history and is not calendar time. Adapted from ref. 1.

patient with liver cirrhosis who developed hepatocellular carcinoma (HCC), novel autoantibodies were detected during the transition period from cirrhosis to HCC (10). The target antigen was identified as a nuclear protein of $64 \mathrm{kDa}$ with structural motifs relegating it to a family of alternative splicing factors. Its tumor-associated function was unknown until 15 years later, when it was shown to inhibit the transformation function of a potent oncoprotein called v-Rel (11). Autoantibodies to TAAs are being reported with increasing frequency, but it is important to be mindful that not all auto- antibodies found in cancer patients are relevant to carcinogenesis. The minimum criteria should be that the presumptive TAAs are involved in tumorigenesis pathways and the autoantibodies have been rigorously shown to be present at significantly higher frequency in cancer than in well-chosen and sufficient non-cancer controls (12).

The immune system leaves imprints of its function in the form of information about the targets of its antibody response. Defining the nature of the target antigens has given us insight into the identity of antigens in immune complexes and the realization that autoantibodies are also useful immunodiagnostic markers. Researchers are now attempting to elucidate the factors that drive the specificity of autoimmune responses.

Address correspondence to: Eng M. Tan, The Scripps Research Institute, 10550 N. Torrey Pines Road, La Jolla, California 92037, USA. Fax: 858.784.2131; E-mail: emtan@scripps.edu.

1. Tan EM, Schur PH, Carr RI, Kunkel GH. Deoxybonucleic acid (DNA) and antibodies to DNA in the serum of patients with systemic lupus erythematosus. JClin Invest. 1966;45(11):1732-1740.

2. Benacerraf B, Potter JC, McCluskey RT, Miller F. The pathologic effects of intravenously administered soluble antigen-antibody complexes: II. Acute glomerulonephritis in rats. J Exp Med. 1960;111(2):195-200.

3. Dixon FJ, Feldman JD, Vazquez JJ. Experimental glomerulonephritis. The pathogenesis of a laboratory model resembling the spectrum of human glomerulonephritis. J Exp Med. 1961;113:899-920.

4. Tan EM. Antinuclear antibodies: diagnostic markers for autoimmune diseases and probes for cell biology. Adv Immunol. 1989;44:93-151.

5. Tan EM. Interactions between autoimmunity and molecular and cell biology. Bridges between clinical and basic sciences. J Clin Invest. 1989;84(1):1-6.

6. Tan EM, Kunkel HG. Characteristics of a soluble nuclear antigen precipitating with sera of patients with systemic lupus erythematosus. J Immunol. 1966;96(3):464-471.

7. Lerner MR, Steitz JA. Antibodies to small nuclear RNAs complexed with proteins are produced by patients with systemic lupus erythematosus. Proc Natl Acad Sci U S A. 1979;76(11):5495-5497.

8. Moroi Y, Peebles C, Fritzler MJ, Steigerwald J, Tan EM. Autoantibody to centromere (kinetochore) in scleroderma sera. Proc Natl Acad Sci U S A. 1980; 77(3):1627-1631.

9. Brenner S, Pepper D, Berns MW, Tan EM, Brinkley BR. Kinetochore structure, duplication and distribution in mammalian cells. Analysis by human autoantibodies from scleroderma patients. J Cell Biol. 1981;91(1):95-102.

10. Imai H, Chan EK, Kiyosawa K, Fu XD, Tan EM. Novel nuclear autoantigen with splicing factor motifs identified with antibody from hepatocellular carcinoma. J Clin Invest. 1993;92(5):2419-2426.

11. Dutta J, Fan G, Gelinas C. CAPER alpha is a novel Rel-TAD- interacting factor that inhibits lymphocyte transformation by the potent Rel/NF-kB oncoprotein v-Rel. J Virol. 2008;82(21):10792-10802.

12. Tan EM, Zhang J. Autoantibodies to tumor-associated antigens: reporters from the immune system. Immunol Rev. 2008;222:328-340. 